\title{
Inducible miR-145 expression by HIF-1 $\alpha$ protects cardiomyocytes against apoptosis via regulating SGK1 in simulated myocardial infarction hypoxic microenvironment
}

\author{
Nianzi Sun ${ }^{1}$, Fanyan Meng ${ }^{2}$, Ning Xue ${ }^{3}$, Guanghui Pang ${ }^{1}$, Qingxin Wang ${ }^{1}$, Hongliang $\mathrm{Ma}^{1}$ \\ ${ }^{1}$ Department of Cardiac Surgery, Linyi People's Hospital, Linyi, China \\ ${ }^{2}$ Operating Room, Linyi People's Hospital, Linyi, China \\ ${ }^{3}$ Qingdao Municipal Health and Family Planning Science and Technology Education Center, Qingdao, China
}

\begin{abstract}
Background: Myocardial infarction (MI) is partly due to myocardial cell damage caused by hypoxia. MicroRNAs (miRNAs) have been proved to be closely related to the development and progression of many cardiovascular diseases. This study investigated the role of miR-145 in cardiomyocytes under hypoxic condition.

Methods: The quantitative real-time polymerase chain reaction ( $q R T-P C R$ ) was performed to test miR-145 expression in H9c2 cells with hypoxia-inducible factor (HIF)- $\alpha$ abnormal expression under hypoxic condition. The 3-(4, 5-dimethyl-2-thiazolyl)-2, 5-diphenyltetrazolium bromide (MTT), Transwell assay and flow cytometry were used to investigate the effects of miR-145 on cell viability, migration and apoptosis under normoxic or hypoxic condition, respectively. Meanwhile, reactive oxygen species (ROS) content in hypoxic H9c2 cells was analyzed. Western blotting was used to explore the potential mechanism of miR-145 protective effects on cardiomyocytes. Expression levels of miR-145 and SGK1 in rat MI model were also assessed.

Results: Results showed that miR-145 was upregulated in H9c2 and HL-1 cells under hypoxic condition, which was promoted by HIF-1 $\alpha$. MiR-145 overexpression enhanced cell viability and migration under normoxic condition. Under hypoxic condition, miR-145 overexpression promoted cell viability, inhibited apoptosis and ROS activity. Western blotting results proved that miR-145 overexpression inhibited the activation of apoptotic related factors, and promoted activation of PI3K/AKT signaling pathway via SGK1 upregulation. Expression levels of miR-145 and SGK1 were both upregulated in rat MI models.

Conclusions: HIF-1 $\alpha$ could induce miR-145 upregulation in hypoxic H9c2 and HL-1 cells. MiR-145 protected H9c2 cells against hypoxic damage. SGK1 upregulation and activated PI3K/AKT may have participated in the protective effects of miR-145 on cardiomyocytes. (Cardiol J 2018; 25, 2: 268-278)

Key words: miR-145, myocardial infarction, hypoxia, HIF-1 $\alpha$, SGK1, PI3K/AKT
\end{abstract}

\section{Introduction}

Myocardial infarction (MI) is one of the leading causes of morbidity and mortality worldwide. Cardiac hypoxia is recognized as one of the main problems for cardiac repair after MI [1]. The current conventional MI treatments including drug therapy, surgery and interventional therapy, can only alleviate the occurrence of heart failure to some extent. Recently, gene therapy in cardiovas-

Address for correspondence: Dr. Hongliang Ma, Department of Cardiac Surgery, Linyi People's Hospital, No. 27, Jiefang East Road, Linyi, Shandong 276000, China, e-mail: mahongliang1451@126.com 
cular disease have achieved initial results. It is well known that genetic, environmental, and epigenetic factors affect the development of atherosclerosis and MI. Many genetic and epigenetic factors which are related with cardiovascular diseases, such as DNA methylation of Aryl hydrocarbon receptor repressor, interleunkin-1 reporter antagonist gene, inhibitor of kappa B-like protein gen polymorphisms, oxidative stress genes and others were investigated [2-5]. The genetic or epigenetic factors were believed to play important roles in the development of MI, suggesting that molecular level study might aid in the understanding of MI. Many studies have confirmed that microRNAs (miRNAs) expression was aberrant after the acute MI, which were involved in the pathophysiology of MI through post-transcriptional regulation effect [6]. Therefore, miRNAs were expected to be the novel biochemical markers and therapeutic targets for treatment of MI.

MiRNAs are small non-coding RNAs which regulate gene expression by either degradation or translational repression of a target mRNA at the post-transcriptional level. They are implicated in regulating diverse cellular processes, such as proliferation, differentiation, development, and cell death $[7,8]$. Recent reports showed that the levels of individual miRNAs and specific miRNAs signatures were linked to the diagnosis and prognosis for various types of human cancers, including chronic lymphocytic leukemia, breast, stomach, colon, pancreas and bladder cancers and includes other cancers as well $[9,10]$. It has been verified that miRNAs were related with human body microenvironments, such as hypoxia. And many hypoxicregulated miRNAs have been identified [11]. For instance, miR-210, miR-155, miR-372/373, and $\mathrm{miR}-10 \mathrm{~b}$ were all found to be upregulated [12-14], whereas miR-20b and miR-200b were found to be down-regulated in response to hypoxia $[15,16]$. However, the exact roles of miRNAs in MI have as yet not been completely understood.

In recent years, miRNAs have been suggested as candidates in the treatment of MI-induced cardiac remodeling for their ability to regulate the expression levels of genes that govern the process of adaptive and maladaptive cardiac remodeling [17, 18]. One study has confirmed that after MI in mice, miRNAs markedly stimulated cardiac regeneration and almost completed the recovery of cardiac functional parameters [19]. MiRNAs were believed to hold great promise for the treatment of cardiac disease which was caused by cardiomyocyte loss.
This study aimed to investigate the role of miR-145 in cardiomyocytes under hypoxic condition. Here, the miR-145 expression in hypoxic H9c2 and HL-1 cells was tested, and its relationship with hypoxia-inducible factor $1 \alpha$ (HIF- $1 \alpha$ ) expression in $\mathrm{H} 9 \mathrm{c} 2$ cells was assessed. The effects of miR-145 on cell bioactivity, cell response to hypoxia and the underlying mechanisms were also investigated.

\section{Methods}

\section{Cell culture}

The rat cardiomyocyte-derived cell line H9c2 and mouse cardiac muscle cell line HL-1 were obtained from American Type Culture Collection (ATCC, Rockefeller, Maryland, USA). Cells were cultured in high glucose Dulbecco's Modified Eagle's Medium (DMEM, GIBCO) supplemented with $10 \%$ (v/v) fetal bovine serum (FBS, Sijiqing, Hangzhou, China) and incubated under normoxia condition $\left(21 \% \mathrm{O}_{2}\right)$ in a standard $\mathrm{CO}_{2}$ incubator, or hypoxic condition $\left(3 \% \mathrm{O}_{2}\right)$ by using a hypoxic workstation (Ruskinn Technology Limited, West Yorkshire, UK) to analog cells hypoxia.

\section{Quantification of miR-145 expression}

The miR-145 expression was evaluated by quantitative real-time polymerase chain reaction (qRT-PCR) with Power SYBR Fast PCR Master Mix and step-one plus real-time PCR machine (Applied Biosystems, Foster City, CA) as described previously [20]. U6 was used as an internal control. All reactions were performed in duplicate. Expression levels of miRNA were quantified employing the $2^{-\Delta \Delta C t}$ relative quantification methods.

\section{Cell transfection}

The recombinant vector pc-DNA ${ }^{\mathrm{TM}} 3.1^{(+)}$-HIF- $1 \alpha$ with full-length of HIF- $1 \alpha$ cording sequences or empty vector as its negative control (Thermo Fisher Scientific) were transfected into cells, respectively. The siRNA against HIF- $1 \alpha$ was purchased from Santa Cruz Biotechnology (sc35561, Santa Cruz, CA). HIF- $1 \alpha$ antibody (C-19) (sc-8711, Santa Cruz, CA) which was confirmed as no silencing double-stranded RNA, was used as a control for siRNA experiments. SGK1 silenced by SGK1 siRNA (sc-33774, Santa Cruz, CA) was performed by the same experimental protocol [21]. For miRNA transfection, cells were transfected with miR-145 mimic, miR-145 inhibitor and negative miRNA control (scramble) (GenePharma, 
Shanghai, China). All the transfections were performed by using Lipofectamine 3000 (Invitrogen Life Technologies, Carlsbad, CA, USA) according to the manufacturer's instructions.

\section{Cell viability assay}

After transfection with either miR-145 mimic, inhibitor, or SGK1 siRNA, H9c2 cells were exposed to normoxic or hypoxic conditions for $48 \mathrm{~h}$ and then cell viability was assessed by using a 3-(4, 5-dimethyl-2-thiazolyl)-2, 5-diphenyltetrazolium bromide (MTT) colorimetric assay according to the standard method. In brief, cells were seeded in 96-well culture plates and incubated for 1-4 days. Then MTT $(20 \mu \mathrm{L} /$ well, Sigma, St. Louis, MO, USA) was added to each well. Cells were incubated for another $4 \mathrm{~h}$ at $37^{\circ} \mathrm{C}$. Then $150 \mu \mathrm{L}$ of dimethyl sulfoxide (DMSO, Sigma, USA) was added to each well and the plates were shaken for $10 \mathrm{~min}$. Each experiment was performed in triplicate. The absorbance of each well was measured at $570 \mathrm{~nm}$ $\left(\mathrm{OD}_{570}\right)$ with a 680 microplate enzyme-linked immunosorbent assay (ELISA) reader (Bio-Rad Laboratories, Hemel Hempsted, UK).

\section{Cell migration assay}

Under normoxic conditions, H9c2 cells migration was determined after transfection with miR-145 mimic or inhibitor by using a modified two-chamber with $8.0 \mu \mathrm{m}$ pore membranes (Greiner 662638). After transfection, cells were resuspended in $200 \mu \mathrm{L}$ of serum-free culture medium and seeded onto the upper compartments of 24-well transwell culture chamber. The low compartment was added $600 \mu \mathrm{L}$ of complete culture medium. Then cells were incubated at $37^{\circ} \mathrm{C}$ for $12 \mathrm{~h}$ and fixed by $4 \%$ methanol (NIST, USA) for $30 \mathrm{~min}$. Non-transferred cells were removed from the upper surface of the filter carefully with a cotton swab. Traversed cells in the lower were stained with $0.1 \%$ crystal violet for 20 min and counted under an optical microscope (Leica Microsystems, Wetzlar, Germany).

\section{Cell apoptosis assay}

Flow cytometry analysis was performed to identify and quantify the apoptotic cells after miR-145 transfection under hypoxic condition, following assay by using Annexin V-FITC/PI apoptosis detection kit (Beijin Biosea Biotechnology, Beijing, China) according to manufacturer's recommendations. After $48 \mathrm{~h}$ of hypoxia administration, cells were collected, washed with PBS and then incubated with $10 \mu \mathrm{L}$ of Annexin V-FITC and $5 \mu \mathrm{L}$ of PI for $30 \mathrm{~min}$ at room temperature in the dark. Apoptotic cells were measured by Epics XL flow cytometer (Beckman Coulter, USA) [22].

\section{Reactive oxygen species (ROS) assay}

The miR-145 modified cells under hypoxic condition were seeded on 6 -well plates, washed twice with PBS, and cultured in serum-free culture medium with $10 \mu \mathrm{M}$ 2, 7-dichlorofluorescein diacetate (DCFH-DA) for $20 \mathrm{~min}$ at $37^{\circ} \mathrm{C}$ under dark conditions. Then cells were collected and suspended within $500 \mu \mathrm{L}$ PBS, and fluorescent intensities were measured by using a flow cytometer at $488 \mathrm{~nm}$ excitation and $521 \mathrm{~nm}$ emission [23].

\section{Rat MI model}

Surgical MI was induced in 6 week old male Sprague-Dawley rats (Charles River Laboratories International. Inc., Wilmington, MA, USA). Briefly, rats were anesthetized with isoflurane, intubated and ventilated with a rodent ventilator (Euthanex Corporation, Allentown, PA, USA). The left anterior descending coronary artery was ligated with silk ligature at a point $1-2 \mathrm{~mm}$ distal to the edge of the left atrium. Evidence of MI was confirmed by ST segment elevation and the appearance of $\mathrm{Q}$ wave on an electrocardiogram. After surgery, rats were randomly assigned into two experimental groups: sham operated (rat chest opened/closed without ligation, $\mathrm{n}=5$ ); MI (rat chest opened/ closed with ligation, $n=5$ ). The animals were then placed on a heating pad and observed every $15 \mathrm{~min}$ until fully recovered from anesthesia as indicated by the ability to maintain sternal recumbency and moving normally. All animal handing protocols were approved by the Institutional Animal Care and Use Committee of Linyi People's Hospital. Buprenorphine was given to the animals before surgery to prevent residual pain during surgery and after surgery to relieve pain and stress during recovery from anesthesia. Each animal was allowed a minimum of 4 weeks of recovery. The animals were then euthanized by an overdose of $\mathrm{Kcl}(2 \mathrm{mg} / \mathrm{kg})$ injection via the tail vein under gas anesthesia. Frozen left ventricular samples used for miR-145 or SGK1 expression level analysis were homogenized in Trizol (Invitrogen Life Technologies, Strathclyde, UK) according to the manufacturer's instructions.

\section{Western blot assay}

The protein expression levels in miR-145 and/or SGK1 siRNA modified H9c2 cells in this 


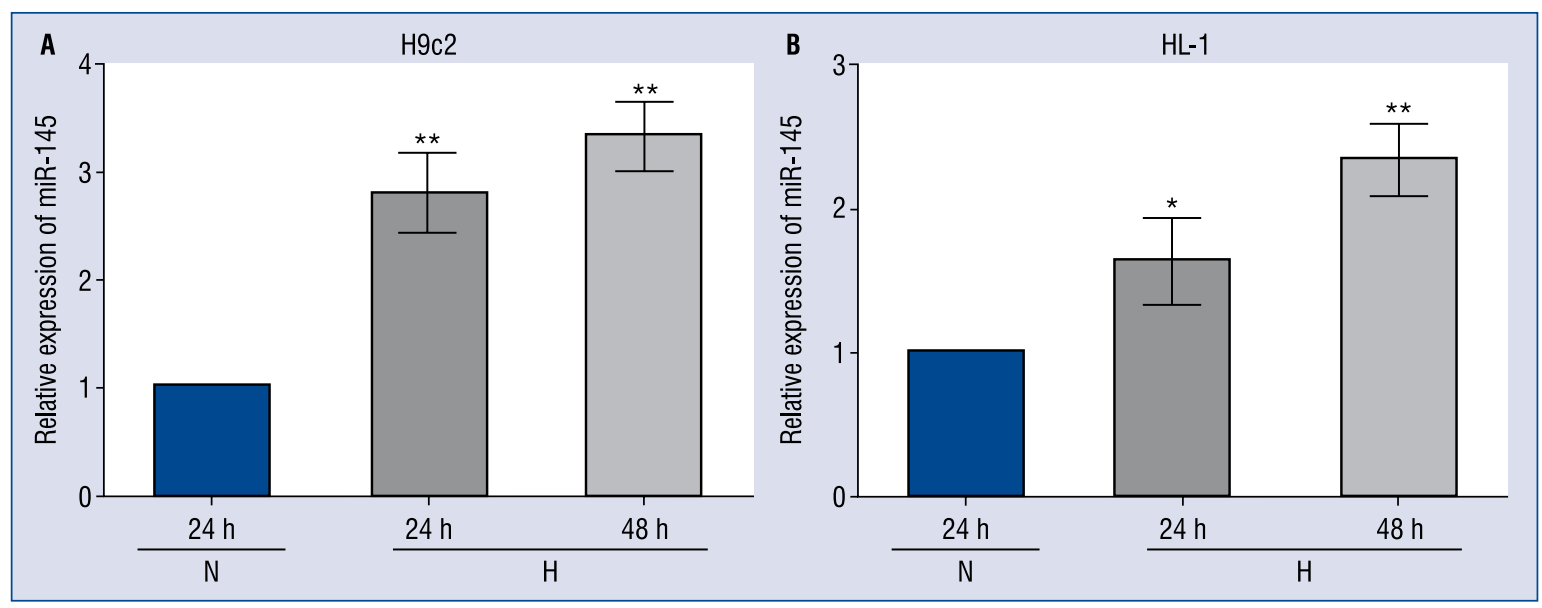

Figure 1. Expression of miR-145 in $\mathrm{H} 9 \mathrm{c} 2$ cells $(\mathbf{A})$ and $\mathrm{HL}-1$ cells $(\mathbf{B})$ which were cultured under normoxic condition $\left(21 \% \mathrm{O}_{2}\right)$ or hypoxic condition $\left(3 \% \mathrm{O}_{2}\right)$ for $24-48 \mathrm{~h}$. The mRNA expression levels of miR-145 were measured by qRT-PCR. U6 acted as an internal control; $\mathrm{N}$ - normoxia; $\mathrm{H}$ - hypoxia; qRT-PCR - quantitative real-time polymerase chain reaction; ${ }^{*} \mathrm{p}<0.05 ;{ }^{*} \mathrm{p}<0.01$.

study were detected by Western blotting. The protein samples were extracted by lysis buffer (Beyotime Biotechnology, Shanghai, China) supplemented with protease inhibitors (Roche, Guangzhou, China), and the $\mathrm{BCA}^{\mathrm{TM}}$ Protein Assay Kit (Pierce, Appleton, WI, USA) was used for protein quantification. Then equal amounts of samples were separated by $10 \%$ sodium dodecyl sulfatepolyacrylamide gel electrophoresis (SDS-PAGE) gels and then transferred onto PVDF membranes (Millipore, Billerica, MA, USA) as described previously [24]. Specific antibodies against HIF-1 $\alpha$ (ab179483); Caspase-9 (ab2014); cleaved Caspase-9 (ab2325); Caspase-3 (ab13847); cleaved Caspase-3 (ab49822); poly ADP ribose polymerase (PARP, ab32138); cleaved-PARP (ab32064); B-cell lymphoma-2 (Bcl-2, ab32124); serum and glucocorticoid-regulated kinase 1 (SGK1, ab59337); PI3K (ab189403); phosphorylated PI3K (p-PI3K, ab182651); AKT (ab8805); phosphorylated AKT (p-AKT, p308, ab38449; p473, ab81283); glyceraldehyde-3-posphate dehydrogenase (GAPDH) (ab8245) (all 1:1,000, Abcam, USA) were used along with appropriate fluorescent secondary antibodies (Santa Cruz biotech).

\section{Statistical analysis}

Data were presented as mean \pm standard deviation (SD). The significance of differences in mean values within and between multiple groups was evaluated using an ANOVA followed by a Duncan's multiple range test. Student's t-test was used to evaluate statistical significance of differences between two groups. $\mathrm{P}<0.05$ was considered statistically significant.

\section{Results}

\section{MiR-145 expression was up-regulated in hypoxic cells}

The miR-145 expression assay was performed by qRT-PCR and results showed that miR-145 expression was significantly increased with time in both H9c2 (Fig. 1A) and HL-1 cells (Fig. 1B) under hypoxic condition ( $3 \%$ oxygen), compared with the normal group $(\mathrm{p}<0.05$ or $\mathrm{p}<0.01)$. It suggested that hypoxia could induce miR-145 upregulation and this induction for miR-145 expression might be in a time-dependent manner.

\section{HIF-1 $\alpha$ promoted miR-145 expression in hypoxic $\mathrm{H} 9 \mathrm{c} 2$ cells}

Hypoxia-induced miRNA expressions have been reported to be associated with HIF- $1 \alpha$ activity in several cell lines [25, 26]. Here, the relationship between miR-145 expression and HIF- $1 \alpha$ exogenous expression or gene silence was analyzed. As shown in Figure 2A and 2B, efficiency of HIF- $1 \alpha$ up-regulation and inhibition were confirmed by Western blot analysis compared with the trace amounts of HIF- $1 \alpha$ expression under normoxic condition. More importantly, miR-145 expression was positively related with HIF- $1 \alpha$ expression under hypoxic condition ( $\mathrm{p}<0.05$, Fig. $2 \mathrm{C}$ ). It suggested that in hypoxic H9c2 cells, miR-145 expression was positively regulated by HIF- $1 \alpha$. 


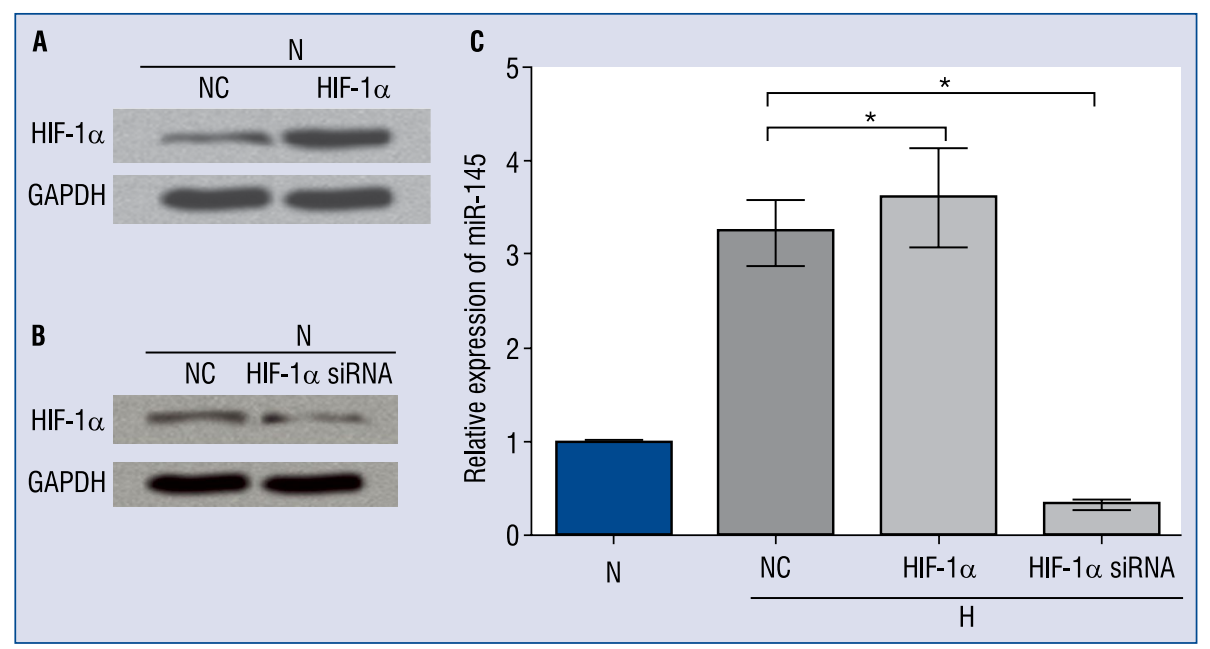

Figure 2. Expression of miR-145 was positively related with HIF-1 $\alpha$ expression in $\mathrm{H} 9 \mathrm{c} 2$ cells under hypoxic condition. A. The HIF-1 $\alpha$ expression in H9c2 cells transfected with recombinant vector pc-DNA ${ }^{\mathrm{TM}} 3.1^{(+)}-\mathrm{HIF}-1 \alpha$ under normoxic condition; B. Expression of HIF-1 $\alpha$ in $\mathrm{H} 9 \mathrm{c} 2$ cells after transfection with HIF-1 $\alpha$ siRNA under normoxic condition. Glyceraldehyde-3-posphate dehydrogenase (GAPDH) acted as an internal control; C. Expression levels of miR-145 in $\mathrm{H} 9 \mathrm{c} 2$ cells after transfection with heterogeneous overexpression of HIF- $1 \alpha$ or HIF-1 $\alpha$ siRNA under normoxic or hypoxic condition were measured by qRT-PCR. U6 acted as an internal control; $\mathrm{N}$ - normoxia; $\mathrm{H}-$ hypoxia for $48 \mathrm{~h}$; NC - HIF- $1 \alpha$ negative control; qRT-PCR - quantitative real-time polymerase chain reaction; ${ }^{*} p<0.05$.

miR-145 enhanced cell viability and migration of $\mathrm{H} 9 \mathrm{c} 2$ cells

First, to determine the efficiency of miR-transfection, the expression of miR-145 in H9c2 cells after transfection with miR-145 mimic, miR-145 inhibitor, or scramble control were measured. The results in Figure 3A showed that transfection with miR-145 mimic increased the expression levels of miR-145 in H9c2 cells under normoxic condition, and miR-145 expression was decreased in the miR-145 inhibitor transfected H9c2 cells.

Under normoxic condition, MTT results (Fig. 3B) showed that after transfection with miR145 mimic, the H9c2 cell viability were significantly increased compared to the control group after 3 and 4 days $(\mathrm{p}<0.01)$. However, transfection with miR-145 inhibitor decreased cell viability $(\mathrm{p}<0.05$ or $\mathrm{p}<0.01$ ). The results of migration assay (Fig. 3C, D) showed that, cell migration was significantly elevated after miR-145 mimic transfection $(\mathrm{p}<0.05)$, while it was decreased by miR-145 inhibitor transfection $(\mathrm{p}<0.05)$ under normoxic condition. These results suggested that miR-145 expression level was positively related with $\mathrm{H} 9 \mathrm{c} 2$ cell viability and migration under normoxic condition.
miR-145 enhanced cell viability and migration while inhibited apoptosis of hypoxic $\mathrm{H} 9 \mathrm{c} 2$ cells

As shown in Figure 4A, H9c2 cells viability was reduced under hypoxic condition. Following with miR-145 mimic transfection, the cells viability was significantly increased $(\mathrm{p}<0.01)$, whereas it was decreased after miR-145 inhibitor transfection compare with negative control $(\mathrm{p}<$ 0.05). ROS assay results (Fig. 4B) suggested that in miR-145 mimic treated cells, the content of ROS was reduced compared with negative control $(\mathrm{p}<$ 0.05 ), while miR-145 inhibitor increased the ROS content $(\mathrm{p}<0.05)$. The flow cytometry analysis results in Figure 4C showed that the apoptotic cells rate was significantly decreased after transfection with miR-145 mimic and elevated in miR-145 inhibitor transfected cells compared with negative control under hypoxic condition $(\mathrm{p}<0.05)$. Then, expression levels of apoptotic related factors by Western blotting were examined. As shown in Figure 4D, after hypoxia administration, expression levels of cleaved form of Caspase-9, Caspase-3 and PARP in H9c2 cells were increased. However, their expressions were significantly decreased after miR-145 mimic transfection and increased 


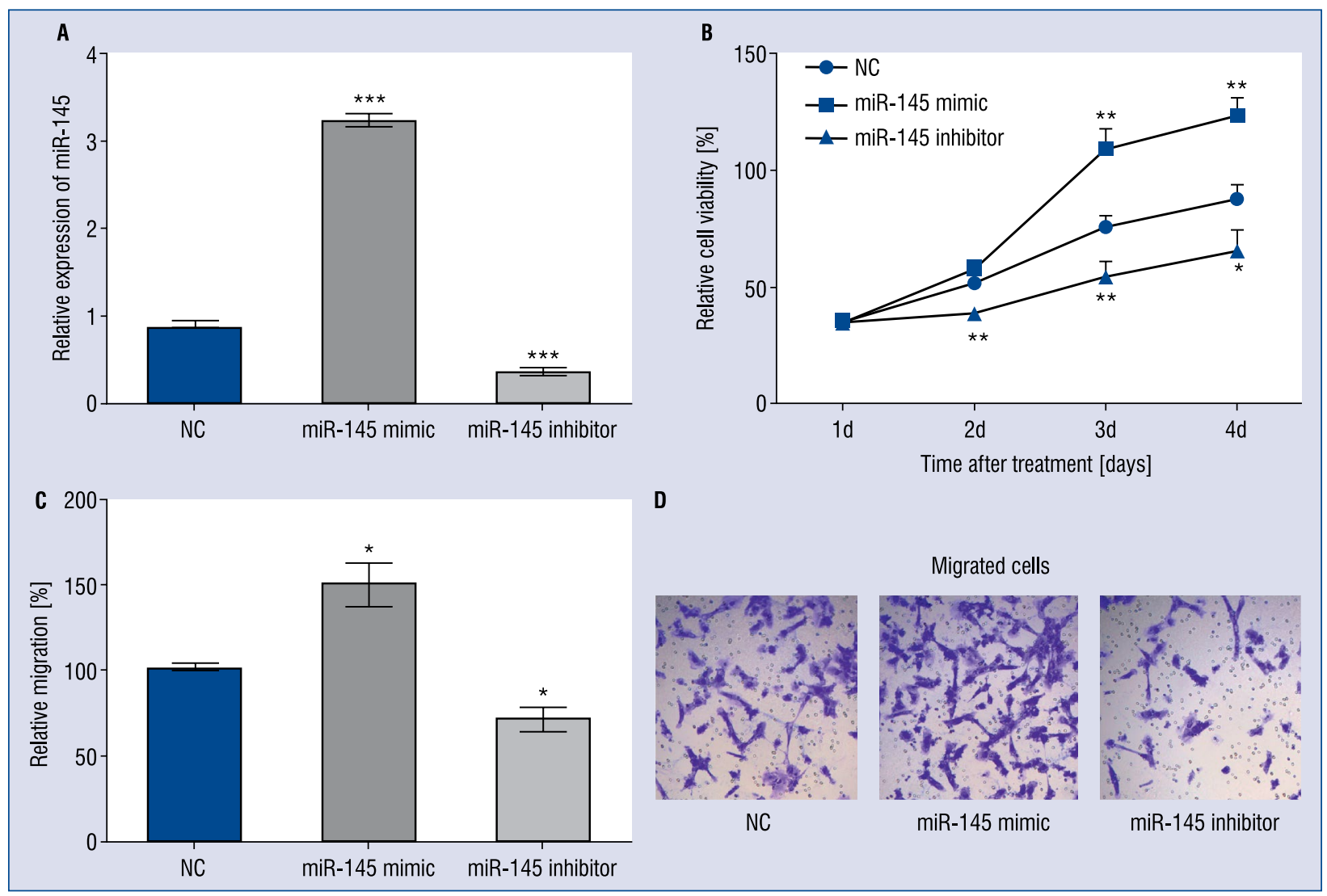

Figure 3. miR-145 upregulation increased cell viability and migration of $\mathrm{H} 9 \mathrm{c} 2$ cells. A. The relative expression of miR145 in H9c2 cells was measured by qRT-PCR. U6 acted as an internal control; B. Cell viability was determined by MTT assay; C. The relative migration of transfected cells was measured by Transwell assay; D. Pictures of cell migration assay results. Transferred cells were strained with $0.1 \%$ crystal violet; $\mathrm{N}-$ normoxia; $\mathrm{H}-$ hypoxia for $48 \mathrm{~h}$; $\mathrm{NC}-$ miR-145 negative control; MTT - 3-(4, 5-dimethyl-2-thiazolyl)-2, 5-diphenyltetrazolium bromide; qRT-PCR - quantative real-time polymerase chain reaction; ${ }^{*} \mathrm{p}<0.05 ;{ }^{*} \mathrm{p}<0.01 ;{ }^{* *} \mathrm{p}<0.001$.

by miR-145 inhibitor transfection. The changes in Bcl-2 expressions were reversed and compare with these three factors above after miR-145 transfections. It suggested that miR-145 could inhibit the apoptosis of hypoxic H9c2 cells. Taken together, these data indicate that miR-145 could affect H9c2 cells bioactivity and protect $\mathrm{H} 9 \mathrm{c} 2$ cells against the hypoxia injury.

\section{MiR-145 was positively related with} the expression of SGK1 and the activation of PI3K and AKT

To further investigate the protective effects of miR-145 on H9c2 cells under hypoxic condition, changes of SGK1 were detected by Western blotting. MiR-145 mimic transfection showed the upregulation effect on SGK1 expression in H9c2 cells under both normoxic and hypoxic conditions, while miR-145 inhibitor transfection decreased expression of SKG1 compared with that in the negative control (Fig. 5A, B). In addition, results in Figure $5 \mathrm{C}$ showed that simultaneous transfection with miR-145 mimic and SGK1 siRNA inhibited SGK1 and Bcl-2 expressions under hypoxia, while increased cleaved-Caspase-3, cleaved-Caspase- 9 and cleaved-PARP expressions compared with miR-145 mimic transfection alone. It suggested that the effects of miR-145 on H9c2 cells under hypoxic condition might be at least in part via modulation of SGK1 and thus protecting H9c2 cells against apoptosis.

To further investigate the potential mechanisms about miR-145 protective effect, expression levels of p-AKT, p-PI3K and Bcl-2 after miR-145 mimic transfection alone, or co-transfection with SGK1 siRNA under normoxic condition were also detected. As shown in Figure 5D, miR-145 mimic transfection increased the expression of SGK1, and also increased the phosphorylation levels of AKT and PI3K. However, after SGK1 siRNA transfec- 


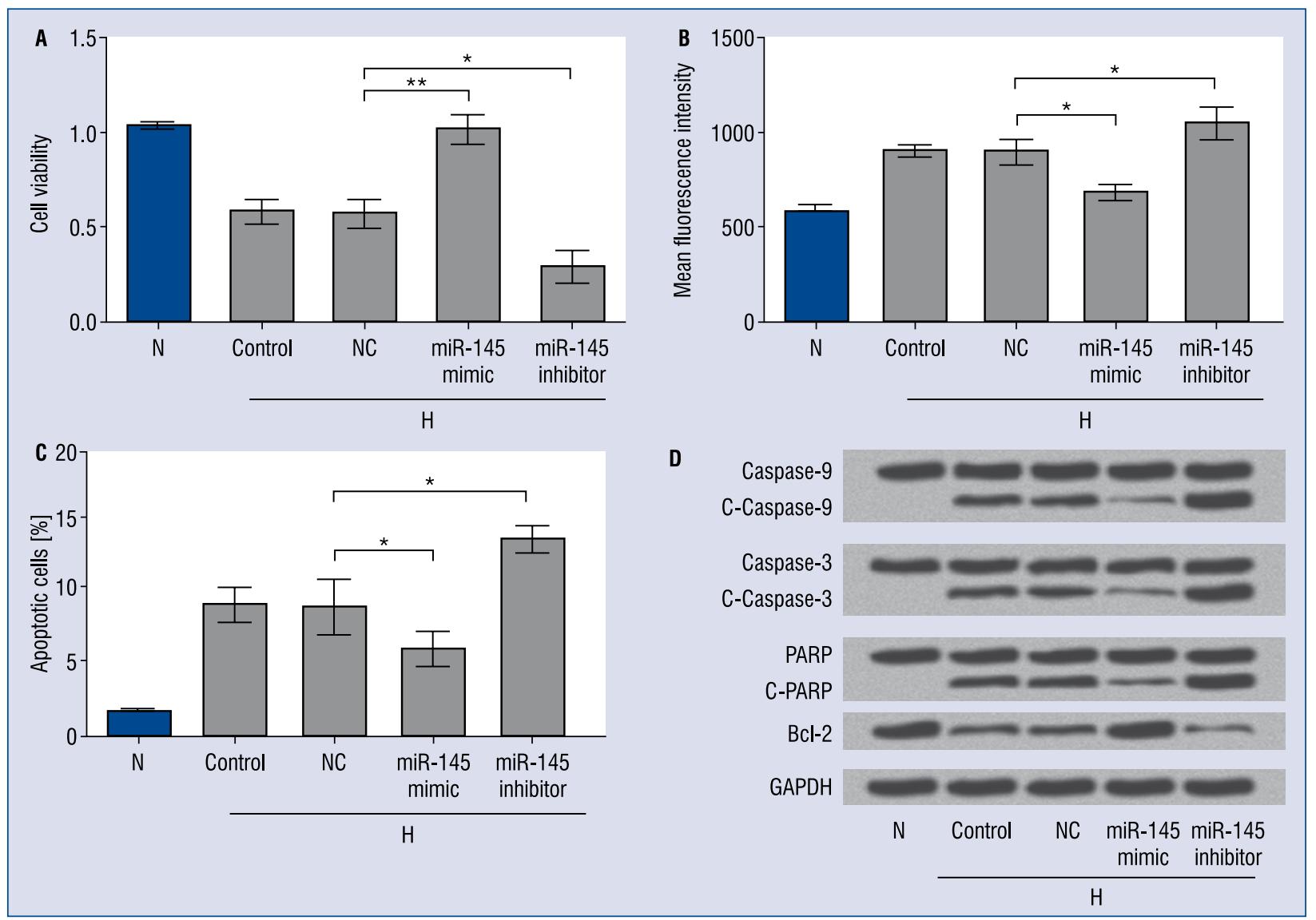

Figure 4. Effects of miR-145 on H9c2 cells under normoxic or hypoxic condition after transfection with miR-145 mimic or miR-145 inhibitor; A. Cell viability was determined by MTT assay; B. Quantitative analysis of reactive oxygen species content by measuring the fluorescent intensities of 2, 7-dichlorofluorescein diacetate; $\mathbf{C}$. The apoptotic cells rate was detected by flow cytometry; D. Expression of apoptosis related factors were measured by Western blotting; $\mathrm{N}$ - normoxia; $\mathrm{H}$ - hypoxia for $48 \mathrm{~h}$; GAPDH - glyceraldehyde-3-posphate dehydrogenase; PARP — poly ADP ribose polymerase; NC - miR-145 negative control; ${ }^{*} \mathrm{p}<0.05 ;{ }^{* *} \mathrm{p}<0.01$.

tion, even with miR-145 mimic transfection, the phosphorylated AKT and PI3K expressions were decreased, as well as the changes of Bcl-2 expressions. Therefore, it could be be infered that SGK1 and PI3K/AKT signaling pathway might also be related with the protective effect of miR-145 on hypoxic H9c2 cells.

Expression levels of miR-145 and SGK1 were significantly increased in ischemia models in vivo

To verify the regulation mechanism of miR-145 in $\mathrm{H} 9 \mathrm{c} 2$ cells under hypoxic condition, the in vivo detections about SGK and miR-145 expression levels were also performed by using a rat MI model. Results in Figure 6 show that miR-145 expression level was significantly increased in MI models compare with that in sham group $(\mathrm{p}<0.05)$. As shown in Figure 6B and 6C, expression of SGK1 was also increased in both mRNA and protein levels compared with the sham group $(\mathrm{p}<0.01$ for $\mathrm{mRNA}$ level). These results suggested that upregulations of miR-145 and SGK1 were observed in MI models in vivo. Combined with the above results, it suggested that SGK1 signaling might be parallel with miR-145 effect on MI in vivo.

\section{Discussion}

In this study, simulative hypoxic myocardial model in vitro by using $\mathrm{H} 9 \mathrm{c} 2$ cell line was constructed, and it was found that miR-145 expression was increased by HIF- $1 \alpha$ overexpression. Meanwhile, miR-145 mimic transfection promoted cell viability and migration, while inhibited apoptosis under hypoxic condition, suggesting that miR-145 might protect $\mathrm{H} 9 \mathrm{c} 2$ cells against hypoxic damage. It was also found that SGK1 and main factors in PI3K/ 


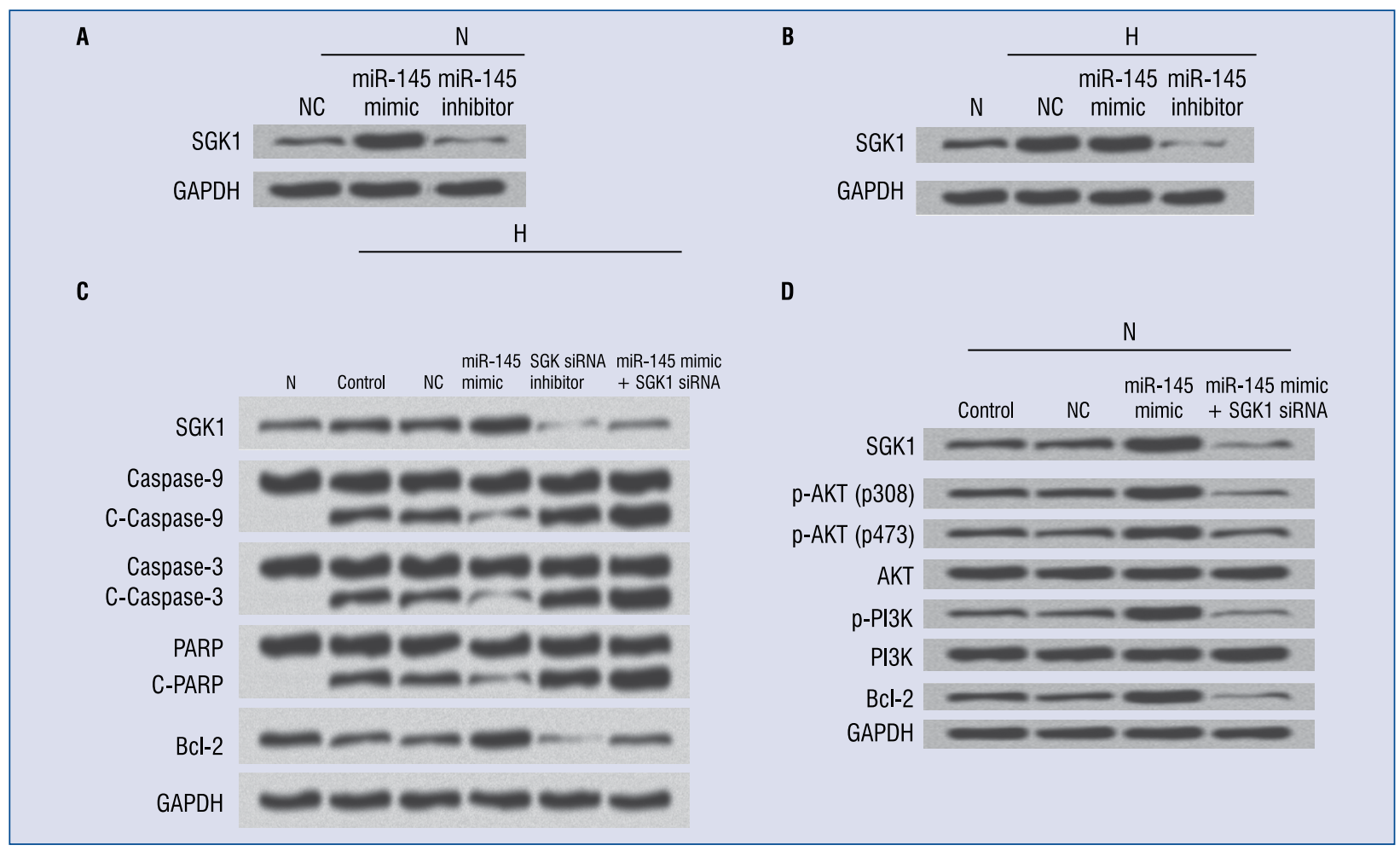

Figure 5. MiR-145 affected SGK1 expression and activation of PI3K/AKT signaling in H9c2 cells. The protein expressions were measured by Western blotting. A. Protein immunofluorescence of SGK1 expression in miR-145 mimic or miR-145 inhibitor transfected H9c2 cells under normoxic condition; B. SGK1 expression in miR-145 transfected H9c2 cells under hypoxic conditions; C. Protein immunofluorescence of apoptosis related factors in $\mathrm{H} 9 \mathrm{c} 2$ cells after been treated with miR-145 mimic and/or SGK1 siRNA under hypoxic condition; D. Protein immunoblots of SGK1, p/t-AKT, $\mathrm{p} / \mathrm{t}-\mathrm{PI} 3 \mathrm{~K}$ and $\mathrm{Bcl}-2$ in $\mathrm{H} 9 \mathrm{c} 2$ cells after the treatments of miR-145 mimic, and/or SGK1 siRNA under normoxic condition. Glyceraldehyde-3-posphate dehydrogenase (GAPDH) acted as an internal control; $\mathrm{N}$ - normoxia; $\mathrm{H}$ - hypoxia for 48 h; NC - miR-145 negative control; C-Caspase-9 — cleaved Caspase-9; C-Caspase-3 — cleaved Caspase-3; PARP — poly ADP ribose polymerase; C-PARP — cleaved PARP; AKT — protein kinase B; $\mathrm{p}-\mathrm{AKT}$ — phosphorylated AKT; $\mathrm{PI} 3 \mathrm{~K}$ - phosphatidylinositol-3 kinase; p-PI3K — phosphorylated PI3K.

/AKT signaling pathway might also be involved in the protective effects of miR-145 on $\mathrm{H} 9 \mathrm{c} 2$ cells under hypoxic condition. MiRNAs regulation has been found to be implicated in many cardiovascular diseases. It has been demonstrated that miRNAs play an important role in the development of pulmonary arterial hypertension which were induced by chronic hypoxia [27]. However, miRNAs regulation and response to microenvironmental factors were relatively little known. These results might provide a basic understanding about the effects of miR-145 on hypoxic cardiomyocytes.

Since it was first reported that the expression of miRNA panel could be induced by hypoxia [28], numerous studies about hypoxia-regulated miRNAs have emerged, including miR-26, miR-107 and miR-210 [29]. Hypoxia is an essential feature of the MI microenvironment, but the molecular mechanisms which are responsible for the hypoxic survival of cardiomyocytes are not fully characterized. It has been shown that hypoxia can leave a specific mark on miRNA profiles in a variety of cell types, with a critical contribution of HIF [28]. Besides, HIF-1 is proved to be a major regulator of hypoxic response after MI [30]. In the present study, compared hypoxic cells with normoxic cells, it was found that hypoxia induced the upregulation of miR-145, and the miR-145 upregulation relied on HIF- $1 \alpha$ expression. These results implied that miR-145 might be a hypoxia-regulated miRNA which was dependent on HIF- $1 \alpha$.

MiR-145, as an important tumor suppressor, shows a significant contribution to tumor pathogenesis in distinct cancer types. It has been proved that miR-145 can induce cancer cell apoptosis and is considered to be an important target for cancer therapy [31, 32]. In the present study, results 

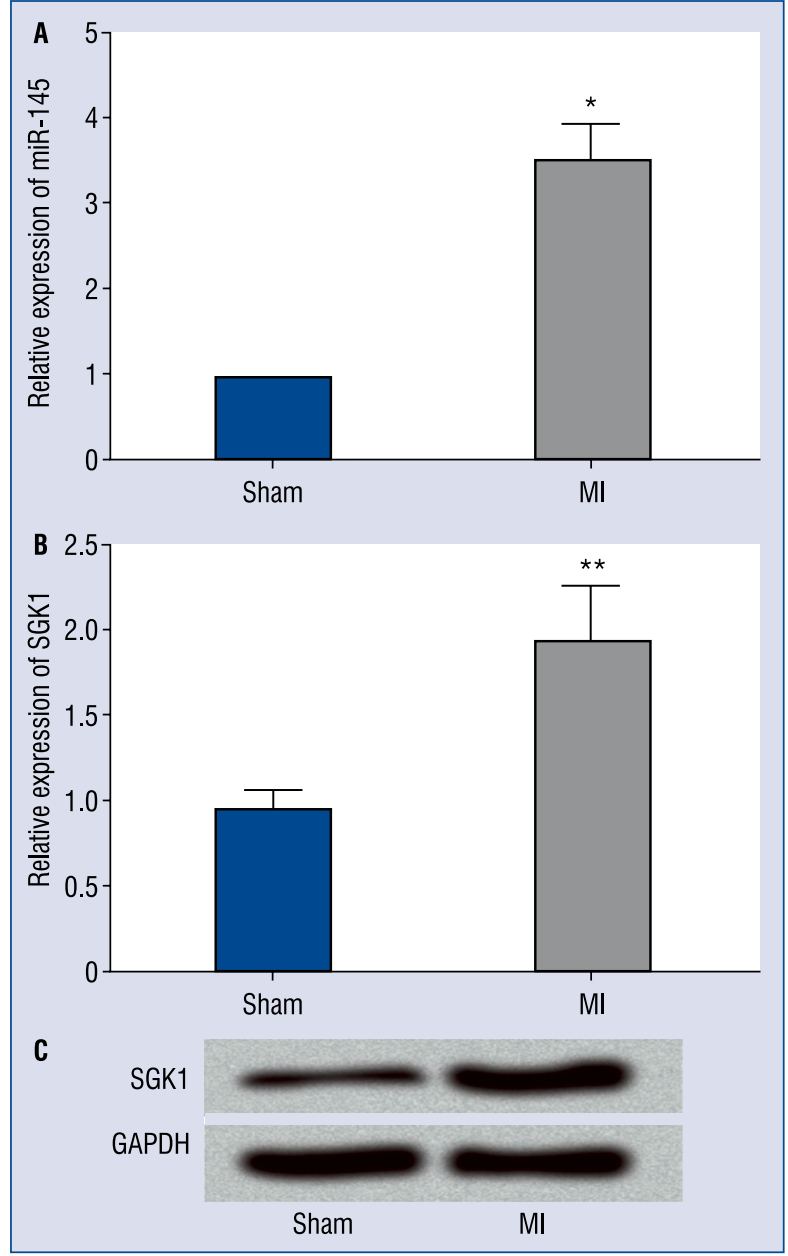

Figure 6. Expression levels of miR-145 and SGK1 were significantly increased in rat $\mathrm{MI}$ model in vivo. The $\mathrm{MI}$ model was established in rats by using ligation on left anterior descending coronary artery. The relative expression levels of miR-145 (A) and SKG1 mRNA (B) in simulated rat $\mathrm{Ml}$ model in vivo were measured by qRT-PCR; C. Protein expression of SGK1 in simulated rat $\mathrm{MI}$ model in vivo was measured by western blotting. Glyceraldehyde-3-posphate dehydrogenase (GAPDH) acted as an internal control. Sham, sham operated (rat chest opened/closed without ligation, $\mathrm{n}=5$ ); MI, myocardial infarction (rat chest opened/closed with ligation, $\mathrm{n}=5$ ); qRT-PCR - quantitative real-time polymerase chain reaction; ${ }^{*} p<0.05 ;{ }^{*} p<0.01$.

suggestthat overexpression of miR-145 promoted $\mathrm{H} 9 \mathrm{c} 2$ cells viability and migration under normoxic condition, as well as under hypoxic condition. MiR-145 overexpression significantly advanced cell viability, reduced apoptosis and damage to $\mathrm{H} 9 \mathrm{c} 2$ cells caused by hypoxia, suggesting that miR-145 could protect H9c2 cells against hypoxia damage, more than a hypoxia-regulated miRNA.
MiR-145 has been proved to be related with apoptosis in urothelial carcinoma cell lines characterized by caspase activation [33]. It was well known that caspase family proteases, are generally an inactive form in normal cells and play an important role in the mechanism of apoptosis [34]. Herein was examined the relationship between miR-145 expression and apoptosis related factors under hypoxic condition. Results showed that miR-145 overexpression inhibited the expression levels of cleaved-Caspase-9, cleaved-Caspase-3 and cleaved-PARP while increased Bcl-2 expression. These results further indicated that miR-145 upregulation might protect cells against cellular apoptosis during hypoxia.

SGK1, as a kinase associated with cell proliferation and apoptosis, is a downstream effector of PI3K implicated in pro-survival mechanisms. According to previous research, inhibition of SGK1 could enhance miRNA-induced apoptosis in cancer cells [35]. Indeed, SGK1 is also shown to enhance colon tumor cell migration via vinculin de-phosphorylation [36]. These findings imply a possible cross connection of SGK1 with miRNA regulation, signaling pathway and cell survival. The present findings showed that miR-145 expression was positively related with SGK1 expression in vitro and in vivo. While combining with the SGK1 inhibition, the miR-145 mimic inhibition effect on expressions of apoptosis related factors were significantly reversed. For the main factors in PI3K/AKT signaling pathway, miR-145 overexpression was positively correlated with the activation of PI3K/AKT signaling, and it might be via SGK1 expression. Thus, the effects of miR-145 on H9c2 cells against hypoxia might be associated with SGK1. SGK1 and PI3K/ /AKT might also be participating in providing a survival advantage to $\mathrm{H} 9 \mathrm{c} 2$ cells.

\section{Conclusions}

In conclusion, it was demonstrated that expression of miR-145 could be regulated by HIF- $1 \alpha$ in cardiomyocytes under hypoxic condition. Further confirmation of the targeting effect of miR-145 could provide strong evidence for miR-145 as a biomarker for clinical diagnosis of MI. In addition, miR-145 upregulation could improve cell survival under hypoxic condition, and inhibited apoptosis via regulating the expressions of apoptosis related factors. SGK1 and PI3K/AKT activation might also be involved in miR-145 protective effects on MI. All these findings implied that hypoxia-induced miR-145 upregulation was related with cell pro- 
tection. It might be worthwhile to validate the miR-145 protective effect against hypoxic-induced injury in the treatment of MI. This study might offer a novel option for early diagnosis of MI.

\section{Conflict of interest: None declared}

\section{References}

1. Lloyd-Jones DA, Carnethon R, Simone M, et al. Heart Disease and Stroke Statistics—2008 Update A Report From the American Heart Association Statistics Committee and Stroke Statistics Subcommittee. Circulation. 2007; 115(5): e25-e146.

2. Starčević JN, Petrovič D. Carotid intima media-thickness and genes involved in lipid metabolism in diabetic patients using statins: a pathway toward personalized medicine. Cardiovasc Hematol Agents Med Chem. 2013; 11(1): 3-8, indexed in Pubmed: 22845899.

3. Kariz S, Milutinović A, Bregar D, et al. The interleukin-1 receptor antagonist gene and the inhibitor of kappa B-like protein gene polymorphisms are not associated with myocardial infarction in Slovene population with type 2 diabetes. Coll Antropol. 2007; 31(2): 503-507, indexed in Pubmed:17847930.

4. Reynolds LM, Wan Ma, Ding J, et al. DNA Methylation of the Aryl Hydrocarbon Receptor Repressor Associations With Cigarette Smoking and Subclinical Atherosclerosis. Circ Cardiovasc Genet. 2015; 8(5): 707-716, doi: 10.1161/CIRCGENETICS.115.001097, indexed in Pubmed: 26307030.

5. Tibaut M, Petrovič D. Oxidative Stress Genes, Antioxidants and Coronary Artery Disease in Type 2 Diabetes Mellitus. Cardiovasc Hematol Agents Med Chem. 2016; 14(1): 23-38, indexed in Pubmed: 27052028.

6. Roy S, Khanna S, Hussain SRA, et al. MicroRNA expression in response to murine myocardial infarction: miR-21 regulates fibroblast metalloprotease-2 via phosphatase and tensin homologue. Cardiovasc Res. 2009; 82(1): 21-29, doi: 10.1093/cvr/ cvp015, indexed in Pubmed: 19147652.

7. Seyhan AA. microRNAs with different functions and roles in disease development and as potential biomarkers of diabetes: progress and challenges. Mol Biosyst. 2015; 11(5): 1217-1234, doi: 10.1039/c5mb00064e, indexed in Pubmed: 25765998.

8. Bartel DP. MicroRNAs: genomics, biogenesis, mechanism, and function. Cell. 2004; 116(2): 281-297, indexed in Pubmed: 14744438 .

9. Taylor DD, Gercel-Taylor C. MicroRNA signatures of tumorderived exosomes as diagnostic biomarkers of ovarian cancer. Gynecol Oncol. 2008; 110(1): 13-21, doi: 10.1016/j.ygyno.2008.04.033, indexed in Pubmed: 18589210.

10. Ferracin M, Negrini M. Micromarkers 2.0: an update on the role of microRNAs in cancer diagnosis and prognosis. Expert Rev Mol Diagn. 2015; 15(10): 1369-1381, doi: 10.1586/14737159.2015.10 81058, indexed in Pubmed: 26338209.

11. McCormick R, Buffa FM, Ragoussis J, et al. The role of hypoxia regulated microRNAs in cancer. Curr Top Microbiol Immunol. 2010; 345: 47-70, doi:10.1007/82_2010_76, indexed in Pubmed: 20549470.

12. Huang X, Ding L, Bennewith KL, et al. Hypoxia-inducible mir210 regulates normoxic gene expression involved in tumor initiation. Mol Cell. 2009; 35(6): 856-867, doi: 10.1016/j.molcel.2009.09.006, indexed in Pubmed: 19782034.
13. Bruning U, Cerone L, Neufeld Z, et al. MicroRNA-155 promotes resolution of hypoxia-inducible factor 1alpha activity during prolonged hypoxia. Mol Cell Biol. 2011; 31(19): 4087-4096, doi: 10.1128/MCB.01276-10, indexed in Pubmed: 21807897.

14. Crosby ME, Kulshreshtha R, Ivan M, et al. MicroRNA regulation of DNA repair gene expression in hypoxic stress. Cancer Res. 2009; 69(3): 1221-1229, doi: 10.1158/0008-5472.CAN-08-2516, indexed in Pubmed: 19141645.

15. Chan YC, Khanna S, Roy S, et al. miR-200b targets Ets-1 and is down-regulated by hypoxia to induce angiogenic response of endothelial cells. J Biol Chem. 2011; 286(3): 2047-2056, doi: 10.1074/jbc.M110.158790, indexed in Pubmed: 21081489.

16. Yuk Ch. Sashwati Roy, and Chandan K. Sen. Regulation of HIF1alpha and VEGF by miR-20b tunes tumor cells to adapt to the alteration of oxygen concentration. J Biol Chem. 2011; 286(3): 2047-2056.

17. Zgheib C, Zouein FA, Kurdi M, et al. Chronic treatment of mice with leukemia inhibitory factor does not cause adverse cardiac remodeling but improves heart function. European Cytokine Network. 2012; 23(4): 191-197.

18. Divakaran V, Mann DL. The emerging role of microRNAs in cardiac remodeling and heart failure. Circ Res. 2008; 103(10): 1072-1083, doi:10.1161/CIRCRESAHA.108.183087, indexed in Pubmed: 18988904.

19. Eulalio A, Mano M, Dal Ferro M, et al. Functional screening identifies miRNAs inducing cardiac regeneration. Nature. 2012; 492(7429): 376-381, doi:10.1038/nature11739, indexed in Pubmed: 23222520 .

20. Ru P, Steele R, Hsueh EC, et al. Anti-miR-203 Upregulates SOCS3 Expression in Breast Cancer Cells and Enhances Cisplatin Chemosensitivity. Genes Cancer. 2011; 2(7): 720-727, doi: 10.1177/1947601911425832, indexed in Pubmed: 22207897.

21. Jiang H, Xiao J, Kang Bo, et al. PI3K/SGK1/GSK3 $\beta$ signaling pathway is involved in inhibition of autophagy in neonatal rat cardiomyocytes exposed to hypoxia/reoxygenation by hydrogen sulfide. Exp Cell Res. 2016; 345(2): 134-140, doi: 10.1016/j. yexcr.2015.07.005, indexed in Pubmed: 26163895.

22. Zhang Ni, Su Y, Xu L. Targeting PKC by miR-143 regulates cell apoptosis in lung cancer. FEBS Lett. 2013; 587(22): 3661-3667, doi:10.1016/j.febslet.2013.09.018, indexed in Pubmed: 24070896.

23. Onoue S, Igarashi N, Yamada S, et al. High-throughput reactive oxygen species (ROS) assay: an enabling technology for screening the phototoxic potential of pharmaceutical substances. J Pharm Biomed Anal. 2008; 46(1): 187-193, doi: 10.1016/j. jpba.2007.09.003, indexed in Pubmed: 17935922.

24. Doiz O, Benito R, Sbihi Y, et al. Western blot applied to the diagnosis and post-treatment monitoring of human hydatidosis. Diagn Microbiol Infect Dis. 2001; 41(3): 139-142, indexed in Pubmed: 11750167.

25. López-Hernández B, Ceña V, Posadas I. The endoplasmic reticulum stress and the HIF-1 signalling pathways are involved in the neuronal damage caused by chemical hypoxia. Br J Pharmacol. 2015; 172(11): 2838-2851, doi: 10.1111/bph.13095, indexed in Pubmed: 25625917.

26. Deng L, Blanco FJ, Stevens H, et al. MicroRNA-143 Activation Regulates Smooth Muscle and Endothelial Cell Crosstalk in Pulmonary Arterial Hypertension. Circ Res. 2015; 117(10): 870-883, doi: 10.1161/CIRCRESAHA.115.306806, indexed in Pubmed: 26311719. 
27. Caruso P, MacLean MR, Khanin R, et al. Dynamic changes in lung microRNA profiles during the development of pulmonary hypertension due to chronic hypoxia and monocrotaline. Arterioscler Thromb Vasc Biol. 2010; 30(4): 716-723, doi: 10.1161/ ATVBAHA.109.202028, indexed in Pubmed:20110569.

28. Kulshreshtha R, Ferracin M, Wojcik SE, et al. A microRNA signature of hypoxia. Mol Cell Biol. 2007; 27(5): 1859-1867, doi: 10.1128/MCB.01395-06, indexed in Pubmed: 17194750.

29. Shen G, Li X, Jia Yf, et al. Hypoxia-regulated microRNAs in human cancer. Acta Pharmacol Sin. 2013; 34(3): 336-341, doi: 10.1038/aps.2012.195, indexed in Pubmed: 23377548.

30. Pugh C. Early expression of angiogenesis factors in acute myocardial. New Engl J Med. 2000; 342(9): 8.

31. Pashaei E, Guzel E, Ozgurses ME, et al. A meta-analysis: identification of common Mir-145 target genes that have similar behavior in different GEO datasets. PLoS One. 2016; 11(9): e0161491, doi: 10.1371/journal.pone.0161491, indexed in Pubmed: 27655328.

32. Matsushita R, Seki N, Yoshino H, et al. Pd38-04 dual tumor-suppressors (Mir-145-5p/mir-145-3p) inducing cancer cell apoptosis via direct targeting Uhrf1 in bladder cancer. J Urol. 2016; 195(4): e924-e925, doi: 10.1016/j.juro.2016.02.1484.

33. Ostenfeld M, Bramsen J, Lamy P, et al. Abstract \#1395: miR-145 expression is reduced in bladder cancer, induces caspase-dependent and -independent cell death, and targets a 22-gene signature that is upregulated in cancer tissue. Cancer Research. 2009: 69.

34. Shakibaei M, John T, Seifarth C, et al. Resveratrol inhibits IL-1 beta-induced stimulation of caspase- 3 and cleavage of PARP in human articular chondrocytes in vitro. Ann N Y Acad Sci. 2007; 1095: 554-563, doi: 10.1196/annals.1397.060, indexed in Pubmed: 17404069.

35. Liu G, Honisch S, Liu G, et al. Inhibition of SGK1 enhances mAR-induced apoptosis in MCF-7 breast cancer cells. Cancer Biol Ther. 2015; 16(1): 52-59, doi: 10.4161/15384047.2014.9869 82, indexed in Pubmed: 25427201.

36. Schmidt EM, Gu S, Anagnostopoulou V, et al. Serum- and glucocorticoid-dependent kinase-1-induced cell migration is dependent on vinculin and regulated by the membrane androgen receptor. FEBS J. 2012; 279(7): 1231-1242, doi: 10.1111/j.17424658.2012.08515.x, indexed in Pubmed:22309306. 